REVISTA MATEMÁTICA COMPLUTENSE

Volumen 11, número 1: 1998

http://dx.doi.org/10.5209/rev_REMA.1998.v11.n1.17328

\title{
Exact controllability of the radial solutions of the semilinear wave equation in $\mathbb{R}^{3}$.
}

\author{
Luz DE TERESA
}

\begin{abstract}
The exact internal controllability of the radial solutions of a semilinear heat equation in $\boldsymbol{R}^{3}$ is proved. The result applies for nonlinearities that are of an order smaller than $|s| \log ^{p}|s|$ at infinity for $1 \leq p<2$. The method of the proof combines HUM and a fixed point technique.
\end{abstract}

\section{Introduction}

In this paper we present a result related with the following general controllability problem for the semilinear wave equation in $\mathbb{R}^{n}$. Let $\Omega$ be a bounded domain of $\mathbb{R}^{n}$ with boundary $\partial \Omega$ of class $C^{2}$. Let $\omega \subset \Omega$, be a nonempty open subset, $\chi_{\omega}$ its characteristic function and $f \in C^{1}\left(\mathbb{R}^{n}\right)$. We consider the following semilinear equation:

$$
\begin{cases}y_{t t}-\Delta y+f(y)=h \chi_{\omega} & \text { in } \Omega \times(0, T) \\ y=0 & \text { on } \partial \Omega \times(0, T) \\ y(x, 0)=y^{0}(x), y_{t}(x, 0)=y^{1}(x) & \text { for } x \in \Omega\end{cases}
$$

where $\left\{y^{0}, y^{1}\right\} \in H_{0}^{1}(\Omega) \times L^{2}(\Omega)$, and $h(x, t) \in L^{2}(\omega \times(0, T))$ is a control function. Suppose that the nonlinearity $f$ is such that the equation has a unique solution

$$
y \in C^{0}\left([0, T] ; H_{0}^{1}(\Omega)\right) \cap C^{1}\left([0, T] ; L^{2}(\Omega)\right) .
$$

A.M.S. Subject Classification: 93B05, 35L05, 35B37.

Servicio Publicaciones Univ. Complutense. Madrid, 1998. 
The problem of exact controllability can be formulated in the following way:

Find conditions on $T$ and $\omega$ (control time and support of the control) such that for every $\left\{y^{0}, y^{1}\right\},\left\{z^{0}, z^{1}\right\} \in H_{0}^{1}(\Omega) \times L^{2}(\Omega)$ we can find a control $h \in L^{2}(\omega \times(0, T))$ such that the corresponding solution $y$ to (1) satisfies the following:

$$
y(x, T)=z^{0}(x), y_{t}(x, T)=z^{1}(x) \text { for } x \in \Omega .
$$

Thus, the question is whether we can drive the system from any initial state to any final state in time $T$ by means of the action of a control with support in $\omega \times(0, T)$. When this property holds true we will say that system (1) is exactly controllable at time $T$.

In the linear framework $(f \equiv 0)$, the problem is by now well understood. In one space dimension with $\omega=\left(l_{1}, l_{2}\right), \Omega=(0,1)$ the exact controllability holds in time $T>2 \max \left(l_{1}, 1-l_{2}\right)$ (see [13]). However, in several space dimensions, the geometric control property is needed on the subset $\omega$ in order to ensure the exact controllability. (See [1].)

When the nonlinearity $f$ is globally Lipschitz there are results of exact controllability in $\mathbb{R}$. In fact, in [14], the exact controllability is proved in several space dimensions when $\omega$ is a neighborhood of the boundary of $\Omega$.

In the one-dimensional case Zuazua [14] proved the existence of $\beta_{0}>0$ such that if

$$
\limsup _{|s| \rightarrow \infty} \frac{|f(s)|}{|s| \log ^{2}|s|}<\beta_{0}
$$

then system (1) is exactly controllable at time $T$ with the same control time that in the linear case. Condition (2) allows nonlinearities that growth at infinity in a superlinear way. This condition is almost optimal since no assumption is done on the sign of the nonlinearity. Indeed, in [11] is proved that if $f$ behaves at infinity like $-s \log ^{p}(|s|)$ with $p>2$, then system (1) is not exactly controllable at any time $T>0$.

The aim of this paper is to adapt the techniques used by Zuazua in [11] to the exact controllability problem for the radial solutions of (1) in $\mathbb{R}^{3}$. 
We shall assume the existence of a constant $C>0$ and $1 \leq p<2$ such that the nonlinearity $f$ satisfies the following grow condition:

$$
\limsup _{|s| \rightarrow \infty} \frac{|f(s)|}{|s|\left|\log ^{p}\right| s||}<C .
$$

Under this assumption, by the methods of Cazenave and Haraux [2], it follows that system (1) has a unique global solution

$$
y \in C^{0}\left([0, T] ; H_{0}^{1}(\Omega)\right) \cap C^{1}\left([0, T], L^{2}(\Omega)\right) .
$$

On the other hand if $y^{0}, y^{1}$ are functions of $|x|, h$ is function of $(|x|, t)$ we obtain that the solution is radial, that is, it depends of $(|x|, t)$. That allows us to transform equation (1), by a change to spherical coordinates, to a semilinear wave equation in $\mathbb{R}$. Nevertheless, working on the space of three-dimensional radial functions introduces new technical difficulties. Observe that these additional difficulties make the grow condition (3), that we are asking to be satisfied by the nonlinearity, to be more restrictive that the condition (2) of the one-dimensional case since we do not reach the critical exponent $p=2$. Nevertheless, this hypothesis improves the result in [10], where we proved the exact controllability of the radial solutions of (1) in $\boldsymbol{R}^{3}$ under the grow condition for the nonlinearity

$$
\limsup _{|s| \rightarrow \infty} \frac{|f(s)|}{|s||\log | s||}<C
$$

for $C$ small enough.

In order to state in a rigorous way the problem, we need to introduce some functional spaces and some of its properties.

Definition 1. Let $\Omega=(0,1) \subset \boldsymbol{R}$

$$
\begin{gathered}
L_{r}^{2}(\Omega)=\left\{f \text { measurable on } \Omega \mid r f(r) \in L^{2}(\Omega)\right\} \\
\|f\|_{L_{r}^{2}}=\left(\int_{0}^{1} r^{2} f^{2} d r\right)^{\frac{1}{2}} \\
H_{r}^{1}(\Omega)=\left\{f \in L_{r}^{2} \text { and } f_{r}=\frac{\partial f}{\partial r} \in L_{r}^{2}\right\} \\
\|f\|_{H_{r}^{1}}=\left(\|f\|_{L_{r}^{2}}^{2}+\left\|f_{r}\right\|_{L_{r}^{2}}^{2}\right)^{1 / 2}
\end{gathered}
$$




$$
\begin{gathered}
L_{r}^{\infty}(\Omega)=\left\{f \text { measurable on } \Omega, \mid r f \in L^{\infty}(\Omega)\right\} \\
\|f\|_{L_{r}^{\infty}}=\|r f\|_{L^{\infty}} \\
V=\left\{f \in H_{r}^{1}(\Omega) \mid f(1)=0\right\}
\end{gathered}
$$

We consider in $V$ the norm induced by $H_{r}^{1}(\Omega)$.

Lemma 1. Let $\Omega=(0,1)$, then

$$
H_{r}^{1}(\Omega) \hookrightarrow L_{r}^{\infty}(\Omega) \text { with compact embedding. }
$$

Proof. Let us see first that $f \in H_{r}^{1}(\Omega)$ implies that $r f \in H^{1}(\Omega)$. It is enough to prove that $f \in L^{2}(\Omega)$ since therefore $(r f)_{r}=f+r f_{r} \in L^{2}(\Omega)$. In fact, this is a consequence of the Hardy inequality that ensures the existence of $C>0$ such that:

$$
\int_{0}^{1} f^{2} d r \leq C\|f\|_{H_{r}^{1}(\Omega)}^{2}
$$

for every $f \in H_{r}^{1}(\Omega)$.

Therefore, the mapping that to $f \in H_{r}^{1}(\Omega)$ associates $r f \in H^{1}(\Omega)$ is continuous. On the other hand, we know that the embedding $H^{1}(\Omega)$ in $L^{\infty}(\Omega)$ is compact. We easily conclude that the embedding $H_{r}^{1}(\Omega)$ in $L_{r}^{\infty}(\Omega)$ is compact.

Remark 1. This lemma allows to prove that the norm of $f$ in $V$ is equivalent to the norm of $f_{r}$ in $L_{r}^{2}(\Omega)$.

\section{Statement of the problem and main result}

Once we have introduced the spaces in which we are going to work, we are in conditions to state our problem. Let $T>0$ and $f \in C^{1}(\mathbb{R})$ satisfying (3).

We put in (1) $\Omega=B_{1} \subset \mathbb{R}^{3}, \omega=B_{l_{2}} \backslash B_{l_{1}}, 0<l_{1}<l_{2} \leq 1$; where $B_{r}$ denotes the ball centered at zero of radius $r$. Assume that $y^{0}, y^{1}$ are functions of $|x|$ and $h$ is function of $(|x|, t)$. We state the problem of exact 
controllability making a change of variables to spherical coordinates $r=$ $|x|$ that transforms the equation in $\mathbb{R}^{3}$ to a wave equation in $\mathbb{R}$. Since the initial data and $h$ depend only of $r$ and of $(r, t)$, respectively, we obtain the following equation for $y$ the solution of (1):

$$
\begin{cases}r^{2} y_{t t}-\left(r^{2} y_{r}\right)_{r}+r^{2} f(y)=r^{2} h \chi_{\omega} & \text { in }(0,1) \times(0, T) \\ y_{r}(0, t)=y(1, t)=0 & \text { for } t \in(0, T) \\ y(r, 0)=y^{0}\left(r^{*}\right), y_{t}(r, 0)=y^{1}(r) & \text { in }(0,1)\end{cases}
$$

where now $\omega=\left(l_{1}, l_{2}\right) \subset \not R, h \in L_{r}^{2}(0,1)$. Since $y$ is the solution of (1) we obtain that $y=y(r, t)$ is the solution of (6) with

$$
y \in C([0, T], V) \cap C^{1}\left([0, T], L_{r}^{2}(0,1)\right) .
$$

The controllability problem is in this case: Find conditions on $T>0$ such that for every

$$
\left\{y^{0}, y^{1}\right\},\left\{z^{0}, z^{1}\right\} \in V \times L_{r}^{2}(0,1)
$$

there exists $h \in L^{2}\left([0, T], L_{r}^{2}(\omega)\right)$ such that the solution $y$ of (6) satisfies

$$
y(r, T)=z^{0}(r), y_{t}(r, T)=z^{1}(r) .
$$

The main result of this paper is the following:

Proposition 1. Let $\omega=\left(l_{1}, l_{2}\right) \neq \emptyset, T>T\left(l_{1}, l_{2}\right)=2 \max \left(l_{1}, 1-l_{2}\right)$. Suppose that $f \in C^{1}(\mathbb{R})$ satisfies

$$
|f(s)| \leq C|s|\left|\log ^{p_{0}}\right| s||+D
$$

for some constants $C, D>0$ and for some $1 \leq p_{0}<2$.

Then, system (6) is exactly controllable in time $T$.

For the proof we adapt the methods introduced by E. Zuazua in [11], where the exact controllability of the semilinear wave equation in $\mathbb{R}$ is proved. First the exact controllability of the linear equation is proved. The proof is based in HUM (Hilbert Uniqueness Method), a method introduced by J.L. Lions [4] to study the exact controllability of linear systems. Chapters 1 and 2 of [4] give a precise description of this method.

Then, the exact controllability of the semilinear wave equation is obtained by a fixed point argument introduced by Zuazua in [14]. This 
fixed point method reduces the exact controllability problem of a semilinear equation to the obtention of suitable a priori estimate for the linear wave equation with a potential.

The rest of the paper is organized as follows. Section 3 is devoted to the development of the fixed point method: Proposition 1 is reduced to the obtention of suitable observability estimates for the wave equation with potential. This estimates are proved in Section 4.

\section{Description of the fixed point method}

In this section we describe the fixed point technique used in the proof of Proposition 1. The proof of Proposition 1 will be reduced to the obtention of a suitable observability property (Proposition 2) for the linear wave equation with a particular potential. This will be done in the next section.

We proceed in several steps.

Step 1 (Linearization).

Let us fix the initial and final data $\left\{y^{0}, y^{1}\right\},\left\{z^{0}, z^{1}\right\} \in V \times L_{r}^{2}(\Omega)$, with $\Omega=(0,1)$, and let us introduce the continuous function

$$
g(s)= \begin{cases}\frac{f(s)-f(0)}{s} & \text { if } s \neq 0 \\ f^{\prime}(0) & \text { if } s=0\end{cases}
$$

We can write (6) as

$$
\begin{cases}r^{2} y_{t t}-\left(r^{2} y_{r}\right)_{r}+r^{2} g(y) y=-r^{2} f(0)+r^{2} h_{\omega} \omega & \text { in } \Omega \times(0, T) \\ y_{r}(0, t)=y(1, t)=0 & \text { for every } t \in(0, T) \\ y(r, 0)=y^{0}(r), y_{t}(r, 0)=y^{1}(r) & \text { in } \Omega .\end{cases}
$$

Then, the proof of Proposition 1 can be reduced to prove the exact controllability of

$$
\begin{cases}r^{2} y_{t t}-\left(r^{2} y_{r}\right)_{r}+r^{2} g(\zeta) y=-r^{2} f(0)+r^{2} h \chi_{\omega} & \text { in } \Omega \times(0, T) \\ y_{r}(0, t)=y^{0}(1, t)=0 & \text { for every } t \in(0, T) \\ y(r, 0)=y^{0}(r), y_{t}(r, 0)=y^{1}(r) & \text { in } \Omega\end{cases}
$$

where $\zeta \in C\left([0, T] ; L_{r}^{\infty}(\Omega)\right)$ and then to obtain a fixed point. 
Remark 2. The bound given by (8) for the nonlinearity $f$ implies, in particular, that $r^{\delta} g(\zeta) \in L^{\infty}(\Omega \times(0, T))$, for every $\delta>0$ and for every $\zeta \in C\left([0, T] ; L_{r}^{\infty}(\Omega)\right)$ :

If $\|\zeta\|_{\infty, r}=0$ we have that $r \zeta=0$ for almost every $r \in \Omega$. Therefore $\zeta(r, t)=0$ for almost every $(r, t)$ and $g(\zeta)=f^{\prime}(0)=k_{1}$ where $k_{1}$ is a constant.

Now, if $\|\zeta\|_{\infty, r} \neq 0$ we use (8) :

$$
\begin{aligned}
\left|r^{\delta} g(\zeta)\right| & \leq C r^{\delta}\left|\log ^{p_{0}}\right| \zeta \|+D \\
& =C r^{\delta}\left|\log ^{p_{0}} \frac{|r \zeta|}{r}\right|+D \\
& \leq C r^{\delta}\left|\log ^{p_{0}} \frac{\|\zeta\|_{L_{r}^{\infty}} \mid+D}{r}\right|+C \\
& \leq C 1 r^{\delta}\left|\log ^{p_{0}}\|\zeta\|_{L_{r}^{\infty}}\right|+C_{2} r^{\delta}\left|\log ^{p_{0}} r\right|+D \\
& \leq C r^{\delta}\left|\log ^{p_{0}}\|\zeta\|_{L_{r}^{\infty}}\right|+D+k_{3} \\
& \leq C_{1}\left|\log ^{p_{0}}\|\zeta\|_{L_{r}^{\infty}}\right|+k_{4} \quad \text { since } r \leq 1
\end{aligned}
$$

The first step is to prove the existence of a control $h=h(r, t ; \zeta) \in$ $L^{2}\left([0, T], L_{r}^{2}(\omega)\right)$ such that the solution $y(r, t ; \zeta)$ of $(10)$ satisfies $(7)$, that is,

$$
y(r, T)=z^{0}(r), y_{t}(r, T)=z^{1}(r) .
$$

To prove this we use HUM.

We first solve the system

$$
\begin{cases}r^{2} z_{t t}-\left(r^{2} z_{r}\right)_{r}+r^{2} g(\zeta) z=-r^{2} f(0) & \text { in } \Omega \times(0, T) \\ z_{r}(0, t)=z(1, t)=0 & \text { for } t \in(0, T) \\ z(r, T)=z^{0}(r), z_{t}(r, T)=z^{1}(r) & \text { in } \Omega\end{cases}
$$

with $\left\{z^{0}, z^{1}\right\} \in V \times L_{\tau}^{2}$. This system has a unique solution

$$
z=z(r, t ; \zeta) \in C([0, T] ; V) \cap C^{1}\left(\{0, T) ; L_{r}^{2}(\Omega)\right)
$$

and it depends continuously on $\left\{z^{0}, z^{1}, \zeta\right\} \in V \times L_{r}^{2}(\Omega) \times L_{r}^{\infty}(\Omega \times(0, T))$. Therefore

$$
z(r, 0, \zeta)=z_{\zeta}^{0} \in V, \quad z_{t}(r, 0, \zeta)=z_{\zeta}^{1} \in L_{r}^{2} .
$$


For any $\left\{\phi^{0}, \phi^{1}\right\} \in L_{r}^{2} \times V^{\prime}$ we solve the system

$$
\begin{gathered}
\begin{cases}r^{2} \phi_{t t}-\left(r^{2} \phi_{r}\right)_{r}+r^{2} g(\zeta) \phi=0 & \text { in } \Omega \times(0, T) \\
\phi_{r}(0, t)=\phi(1, t)=0 & \text { for } t \in(0, T) \\
\phi(r, 0)=\phi^{0}(r), \phi_{t}(r, 0)=\phi^{1}(r) & \text { in } \Omega\end{cases} \\
\begin{cases}r^{2} \nu_{t t}-\left(r^{2} \nu_{r}\right)_{r}+r^{2} g(\zeta) \nu=r^{2} \phi \chi_{\omega} & \text { in } \Omega \times(0, T) \\
\nu_{r}(0, t)=\nu(1, t)=0 & \text { for } t \in(0, T) \\
\nu(r, T)=\nu_{t}(r, T)=0 & \text { in } \Omega .\end{cases}
\end{gathered}
$$

We define the linear and continuous operator

$$
\Lambda_{\zeta}: L_{r}^{2} \times V^{\prime} \rightarrow L_{r}^{2} \times V
$$

by

$$
\Lambda_{\zeta}\left\{\phi^{0}, \phi^{\mathrm{i}}\right\}=\left\{-\nu_{t}(r, 0), \nu(r, 0)\right\} .
$$

The problem is reduced to prove the existence of some $\left\{\phi^{0}, \phi^{1}\right\} \in L_{\tau}^{2} \times V^{\prime}$ such that

$$
\Lambda_{\zeta}\left\{\phi^{0}, \phi^{1}\right\}=\left\{-y^{1}+z_{\zeta}^{1}, y^{0}-z_{\zeta}^{0}\right\}
$$

Indeed, if $\left\{\phi^{0}, \phi^{1}\right\}$ is the solution of (15), then $\nu$, the corresponding solution of (13), satisfies

$$
\nu(0)=y^{0}-z_{\zeta}^{0}, \nu_{t}(0)=y^{1}-z_{\zeta}^{1}
$$

and therefore $y=\nu+z$ satisfies both (10) and (7).

In order to solve (15) we multiply (13) by the solution $\phi$ of (12) and integrating by parts we observe that

$$
-\int_{0}^{1} r^{2} \nu_{t}(r, 0) \phi^{0}+<\nu(r, 0), \phi^{1}>_{V \times V^{\prime}}=\int_{\omega \times(0, T)} r^{2} \phi^{2} d r d t .
$$

That implies that

$$
<\Lambda_{\zeta}\left\{\phi^{0}, \phi^{1}\right\},\left\{\phi^{0}, \phi^{1}\right\}>=\int_{\omega \times(0, T)} r^{2} \phi^{2} d r d t \text { for every }\left\{\phi^{0}, \phi^{1}\right\} \in L_{r}^{2}(\Omega) \times V^{\prime}
$$

where $<$, > denotes the duality pairing between $L_{r}^{2}(\Omega) \times V$ and $L_{r}^{2}(\Omega) \times$ $V^{\prime}$. 
Step 2. Let us assume that the following observability inequality holds for system (12): there exist $q=q(p), 2<q<\infty$ and two positive constants $A, B>0$ such that

$$
\left\|\left\{\phi^{0}, \phi^{1}\right\}\right\|_{L_{r}^{2} \times V^{\prime}}^{2} \leq A e^{B\left\|r^{1 / 2 q} g(\zeta)\right\|_{\infty}^{1 / p}} \int_{\omega \times(0, T)} r^{2} \phi^{2} d r d t
$$

We get

$$
<\Lambda_{\zeta}\left\{\phi^{0}, \phi^{1}\right\},\left\{\phi^{0}, \phi^{1}\right\}>\geq A e^{-B\left\|r^{1 / 2 q} g(\zeta)\right\|_{\infty}^{1 / p}}\left\|\left\{\phi^{0}, \phi^{1}\right\}\right\|^{2}
$$

and therefore

$$
\Lambda_{\zeta}: L_{r}^{2} \times V^{\prime} \rightarrow L_{r}^{2} \times V \text { is an isomorphism. }
$$

Then, equation (15) has a unique solution

$$
\left\{\phi^{0}, \phi^{1}\right\}=\left\{\phi^{0}(r, \zeta), \phi^{1}(r, \zeta)\right\} \in L_{r}^{2} \times V^{\prime} .
$$

Moreover, the solution of (10) corresponding to the control

$$
h=\phi(r, t ; \zeta)
$$

satisfies (7).

We have defined in a unique way a control $h(r, t ; \zeta) \in L^{2}\left([0, T], L_{r}^{2}(\omega)\right)$ for system $(10)-(7)$ and this for every $r \zeta \in L^{\infty}(\Omega \times(0, T))$. The solution $y$ of $(10)$ belongs to $C([0, T] ; V) \cap C^{1}\left([0, T] ; L_{r}^{2}(\Omega)\right)$ and since $H_{r}^{1}(\Omega) \subset L_{r}^{\infty}(\Omega)$ we deduce that $y \in C\left([0, T] ; L_{r}^{\infty}(\Omega)\right)$. Therefore, we have constructed a nonlinear operator

$$
\mathbf{K}: C\left([0, T], L_{r}^{\infty}(\Omega)\right) \rightarrow C\left([0, T], L_{r}^{\infty}(\Omega)\right)
$$

such that $\mathbf{K}(\zeta)=y$ where $y$ is the solution of (10)-(7) with the control function $h \in L^{2}\left((0, T), L_{r}^{2}(\omega)\right)$ defined above.

Since the solutions of $(10)$ depend continuously on $\left\{y^{0}, y^{1}, \zeta, h\right\} \in$ $V \times L_{r}^{2}(\Omega) \times C\left([0, T] ; L_{r}^{\infty}(\Omega)\right) \times L^{2}\left(0, T ; L_{r}^{2}(\omega)\right)$, we have that the operator $\mathrm{K}$ sends bounded sets of $C\left([0, T], L_{\tau}^{\infty}(\Omega)\right)$ into bounded sets of $C([0, T] ; V) \cap C^{1}\left([0, T] ; L_{r}^{2}(\Omega)\right)$.

This fact, combined with the compactness of the embedding $C([0, T] ; V) \cap C^{1}\left([0, T] ; L_{r}^{2}(\Omega)\right) \subset C\left([0, T], L_{r}^{\infty}(\Omega)\right)$ (see Simon $[7]$ Theorem 5$)$ allows us to prove both the continuity of $\mathbf{K}$ and the fact that 
K maps bounded sets of $C\left([0, T], L_{r}^{\infty}(\Omega)\right)$ into relatively compact sets of itself. Therefore the operator

$$
\mathbf{K}: C\left([0, T], L_{r}^{\infty}(\Omega)\right) \rightarrow C\left([0, T], L_{r}^{\infty}(\Omega)\right)
$$

is compact.

Step 3 (Fixed point).

It is enough to prove the existence of a fixed point of $K$. Indeed, if $\zeta=y \in C\left([0, T], L_{r}^{\infty}(\Omega)\right)$ is a fixed point of $\mathbf{K}$, then $\zeta=y \in C([0, T] ; V) \cap C^{1}\left([0, T] ; L_{r}^{2}(\Omega)\right)$ and $y$ satisfies both (6) and (7).

Therefore, if $\zeta=y$ is a fixed point of $\mathbf{K}$, it is sufficient to choose $h(r, t ; \zeta)$ as control for the nonlinear system $(6)$.

In order to prove the existence of a fixed point for $K$, we use the Leray-Shauder's degree theorem. We define the operator

$$
\mathcal{K}:[0,1] \times C\left([0, T], L_{r}^{\infty}(\Omega)\right) \rightarrow C\left([0, T], L_{\tau}^{\infty}(\Omega)\right)
$$

such that

$$
\mathcal{K}(\sigma, \zeta)=\mathbf{K}_{\sigma}(\zeta)
$$

where $\mathbf{K}_{\sigma}$ is the compact operator defined in Step 2 but for the nonlinearity $\sigma g$. The operator $\mathcal{K}$ is compact and $\mathcal{K}(0, \zeta)=\mathbf{K}_{0}(\zeta)$ is independent of $\zeta$. Therefore, in order to conclude the existence of a fixed point for $\mathbf{K}=\mathbf{K}_{1}$ it is enough to prove that the identity

$$
\mathcal{K}\left(\sigma, y_{\sigma}\right)=y_{\sigma}
$$

with $\sigma \in[0,1]$ and $y_{\sigma} \in C\left([0, T], L_{r}^{\infty}(\Omega)\right)$ implies an uniform bound for $y_{\sigma}$ in $C\left([0, T], L_{r}^{\infty}(\Omega)\right)$.

By construction of $\mathcal{K}$, equation (17) is equivalent to the system

$$
\begin{cases}r^{2} y_{\sigma, t t}-\left(r^{2} y_{\sigma, r}\right)_{r}+r^{2} \sigma f\left(y_{\sigma}\right)=r^{2} \phi_{\sigma} \chi_{\omega} & \text { in } \Omega \times(0, T) \\ y_{\sigma, r}(0, t)=y(1, t)=0 & \text { for } t \in(0, T) \\ y_{\sigma}(r, 0)=y^{0}(r), y_{\sigma, t}(r, 0)=y^{1}(r) & \text { in } \Omega \\ y_{\sigma}(r, T)=z^{0}(r), y_{\sigma, t}(r, T)=z^{1}(r) & \text { in } \Omega \\ r^{2} \phi_{\sigma, t t}-\left(r^{2} \phi_{\sigma, r}\right)_{r}+r^{2} g\left(y_{\sigma}\right) \phi_{\sigma}=0 & \text { in } \Omega \times(0, T) \\ \phi_{\sigma, r}(0, t)=\phi_{\sigma}(1, t)=0 & \text { for } t \in(0, T) \\ \phi_{\sigma}(r, 0)=\phi_{\sigma}^{0}, \phi_{\sigma, t}(r, 0)=\phi_{\sigma}^{1}(r) & \text { for } r \in \Omega .\end{cases}
$$


Let us assume for the moment that $f(0)=0$. Multiplying by $\phi_{\sigma}$ the equation satisfied by $y_{\sigma}$ and integrating by parts in $\Omega \times(0, T)$, we get

$$
\begin{aligned}
\int_{\omega \times(0, T)} r^{2} \phi_{\sigma}^{2} d r d t & =\int_{0}^{1} r^{2} z^{1} \phi_{\sigma}(T)-\int_{0}^{1} r^{2} y^{1} \phi_{\sigma}^{0} \\
& -<z^{0}, \phi_{\sigma, t}(T)>_{V, V^{\prime}}+<y^{0}, \phi_{\sigma}^{1}>_{V, V^{\prime}} .
\end{aligned}
$$

From (19) we deduce

$$
\int_{\omega \times(0, T)} r^{2} \phi_{\sigma}^{2} d r d t \leq C_{U}\left(\left\|\left\{\phi_{\sigma}^{0}, \phi_{\sigma}^{1}\right\}\right\|_{L_{r}^{2} \times V^{\prime}}+\left\|\left\{\phi_{\sigma}(T), \phi_{\sigma, t}(T)\right\}\right\|_{L_{r}^{2} \times V^{\prime}}\right)
$$

with $C_{0}=\max \left\{\left\|\left\{y^{0}, y^{1}\right\}\right\| V \times L_{r}^{2},\left\|\left\{z^{0}, z^{1}\right\}\right\|_{V \times L_{r}^{2}}\right\}$.

In view of (16) and by the time-reversibility of the equation satisfied by $\phi_{\sigma}$ we deduce

$$
\begin{aligned}
\left\|\left\{\phi_{\sigma}^{0}, \phi_{\sigma}^{1}\right\}\right\|_{L_{r}^{2} \times V^{\prime}}^{2} & \leq A e^{\sigma B\left\|r^{1 / 2 q} g\left(y_{\sigma}\right)\right\|_{L^{\infty}}^{1 / p}} \int_{\omega \times(0, T)} r^{2} \phi_{\sigma}^{2} d r d t \\
\left\|\left\{\phi_{\sigma}(T), \phi_{\sigma, t}(T)\right\}\right\|_{L_{r}^{2} \times V^{\prime}}^{2} & \leq A e^{B \sigma\left\|r^{1 / 2 q} g\left(y_{\sigma}\right)\right\|_{L^{\infty}}^{1 / p}} \int_{\omega \times(0, T)} r^{2} \phi_{\sigma}^{2} d r d t
\end{aligned}
$$

and we get

$$
\begin{gathered}
\left(\left\|\left\{\phi_{\sigma}^{0}, \phi_{\sigma}^{1}\right\}\right\|_{L_{\tau}^{2} \times V^{\prime}}+\left\|\left\{\phi_{\sigma}(T), \phi_{\sigma, t}(T)\right\}\right\|_{\left.L_{T}^{2} \times V^{\prime}\right)^{2}}\right. \\
\leq C e^{\sigma B\left\|r^{1 / 2 q} g\left(y_{\sigma}\right)\right\|_{L^{\infty}}^{1 / p}} \int_{\omega \times(0, T)} r^{2} \phi_{\sigma}^{2} d r d t .
\end{gathered}
$$

Combining (20) and (21) we get

$$
\begin{aligned}
& \left(\left\|\left\{\phi_{\sigma}^{0}, \phi_{\sigma}^{1}\right\}\right\|_{L_{\sigma}^{2} \times V^{\prime}}+\left\|\left\{\phi_{\sigma}(T), \phi_{\sigma, t}(T)\right\}\right\|_{L_{r}^{2} \times V^{\prime}}\right)^{2} \\
& \leq C e^{\sigma B\left\|r^{1 / 2 q} g\left(y_{\sigma}\right)\right\|_{L^{\infty}}^{1 / p}} \int_{\omega \times(0, T)} r^{2} \phi_{\sigma}^{2} d r d t \\
& \leq C_{0} e^{\sigma B\left\|r^{1 / 2 q} g\left(y_{\sigma}\right)\right\|_{L^{\prime \infty}}^{1 / \infty}}\left(\left\|\left\{\phi_{\sigma}^{0}, \phi_{\sigma}^{1}\right\}\right\|_{L_{\sigma}^{2} \times V^{\prime}}+\left\|\left\{\phi_{\sigma}(T), \phi_{\sigma, t}(T)\right\}\right\|_{L_{\sigma}^{2} \times V^{\prime}}\right)
\end{aligned}
$$

that means that

$$
\left(\left\|\left\{\phi_{\sigma}^{0}, \phi_{\sigma}^{1}\right\}\right\|_{L_{r}^{2} \times V^{\prime}}+\left\|\left\{\phi_{\sigma}(T), \phi_{\sigma, t}(T)\right\}\right\|_{L_{r}^{2} \times V^{\prime}}\right) \leq C_{0} e^{\sigma B\left\|r^{1 / 2 \tau} g\left(y_{\sigma}\right)\right\|_{L^{\prime}}^{1 / D_{0}}} .
$$

Since $\sigma \in(0,1)$

$$
e^{\sigma B\left\|r^{1 / 2 q} g\left(y_{\sigma}\right)\right\|_{\infty}^{1 / p}} \leq e^{B\left\|r^{1 / 2 q} g\left(y_{\sigma}\right)\right\|_{\infty}^{1 / p}}
$$


Now, if $\left\|r y_{\sigma}\right\|_{\infty}>1$, in view of (11) we have

$$
e^{\sigma B\left\|r^{1 / 2 q} g\left(y_{\sigma}\right)\right\|_{\infty}^{1 / p}} \leq e^{C_{1}^{1 / \nu} \beta_{0}\left|\log \left(\left\|r y_{\sigma}\right\|_{\infty}\right)\right|+k_{3} \mid}
$$

where $k_{3}$ is a constant independent of $y_{\sigma}, \sigma$ and $\beta_{0}=\log ^{p_{0} / p-1}\left\|r y_{\sigma}\right\|_{\infty}$. In consequence,

$$
\begin{gathered}
\left(\left\|\left\{\phi_{\sigma}^{0}, \phi_{\sigma}^{1}\right\}\right\|_{L_{r}^{2} \times V^{\prime}}+\left\|\left\{\phi_{\sigma}(T), \phi_{\sigma, t}(T)\right\}\right\|_{L_{r}^{2} \times V^{\prime}}\right) \\
\leq C_{0} e^{C_{1}^{1 / p} \beta_{0} B\left|\log \left(\left\|r y_{\sigma}\right\|_{\infty}\right)\right|+k_{3} \mid}
\end{gathered}
$$

\section{Step 4.}

We need to estimate $\left\|r y_{\sigma}\right\|_{\infty}$ in terms of $\int_{\omega \times(0, T)} r^{2} \phi_{\sigma}^{2} d r d t$. Assume that we have the following estimate (consequence of Lemma 4 in the next section) for $y_{\sigma}$ :

$$
\left\|y_{\sigma}(t)\right\|_{H_{r}^{1}} \leq A\left(\left\|y^{0}\right\|_{V}^{2}+\left\|y^{1}\right\|_{L_{r}^{2}}^{2}+\int_{\omega \times(0, T)} r^{2} \phi_{\sigma^{2}}^{2} d r d t\right) e^{B\left\|r^{1 / 2 q} g\left(y_{\sigma}\right)\right\|_{\infty}^{1 / p_{r}} T} .
$$

By the continuous embedding (4) we deduce that for $C$ large enough

$$
\left\|r y_{\sigma}\right\|_{\infty}^{2} \leq C\left(\left\|y^{0}\right\|_{V}^{2}+\left\|y^{1}\right\|_{L_{r}^{2}}^{2}+\int_{\omega \times(0, T)} r^{2} \phi_{\sigma}^{2} d r d t\right) e^{C\left\|r^{1 / 2 q_{g}} g\left(y_{\sigma}\right)\right\|_{\infty}^{1 / p_{T}}}
$$

which combined with (11), yields

$$
\begin{aligned}
\left\|r y_{\sigma}\right\|_{\infty}^{2} \leq C & \left(\left\|y^{0}\right\|_{V}^{2}+\left\|y^{1}\right\|_{L_{r}^{2}}^{2}+\int_{\omega \times(0, T)} r^{2} \phi_{\sigma}^{2} d r d t\right) \\
& e^{C d T} e^{C T C_{1}^{1 / p} \beta_{0}\left|\log \left(\left\|r y_{\sigma}\right\|_{\infty}\right)\right|}
\end{aligned}
$$

where $\beta_{0}=\log ^{p_{0} / p-1}\left\|r y_{\sigma}\right\|_{\infty}$. Since $p>p_{0}$, there exists $\tilde{C}>0$ such that if $\left\|r y_{\sigma}\right\|_{\infty}>\tilde{C}$ then $C T C_{1}^{1 / p} \beta_{0}<1$. We can assume that $\left\|r y_{\sigma}\right\|_{\infty}>\tilde{C}$ since the contrary will conclude the proof.

We deduce that

$$
\left\|r y_{\sigma}\right\|_{\infty} \leq 1+C\left(\left\|y^{0}\right\|_{V}^{2}+\left\|y^{1}\right\|_{L_{r}^{2}}^{2}+\int_{\omega \times(0, T)} r^{2} \phi_{\sigma}^{2} d r d t\right) e^{C d T} .
$$


Combining (26) with (20) we get

$\left\|r y_{\sigma}\right\|_{\infty} \leq 1+C\left[\left\|y^{0}\right\|_{V}^{2}+\left\|y^{1}\right\|_{L_{F}^{2}}^{2}+C_{0}\left(\left\|\left\{\phi_{a}^{0}, \phi_{a}^{1}\right\}\right\|+\left\|\left\{\phi_{\sigma}(T), \phi_{\sigma, t}(T)\right\}\right\|\right] e^{C d T}\right.$

Let us denote $\mathcal{F}=\left\|\left\{\phi_{\sigma}^{0}, \phi_{\sigma}^{1}\right\}\right\|_{L_{r}^{2} \times V^{\prime}}+\|\left\{\phi_{\sigma}(T), \phi_{\sigma, t}(T) \|_{L_{r}^{2} \times V^{\prime}}\right.$. From (23) and (27) we deduce that

$$
\mathcal{F} \leq D e^{\left(\beta_{0}\left(C C_{1} T\right)^{1 / p} B \log \left[1+C\left(\left\|y^{0}\right\|_{V}^{2}+\left\|y^{1}\right\|_{L_{r}^{2}}^{2}+C_{0} \mathcal{F}\right) e^{C d T}\right]+k_{3}\right)}
$$

where $D, C_{0}, C, k_{3}$ are positive constants independent of $\sigma$. Again, we get that $\beta_{0}\left(C C_{1} T\right) B<1$ for $\left\|r y_{\sigma}\right\|_{\infty}$ large enough. We deduce that $\left(\left\|\left\{\phi_{\sigma}^{0}, \phi_{\sigma}^{1}\right\}\right\|+\left\|\left\{\phi_{\sigma}(T), \phi_{\sigma, t}(T)\right\}\right\|\right)$ is uniformly bounded, which combined with (27) yields an uniform bound for $\left\|r y_{\sigma}\right\|_{\infty}$.

\section{Step 5.}

Let us return to the case $f(0) \neq 0$. Instead of (19) we have

$$
\begin{aligned}
\int_{\omega \times(0, T)} r^{2} \phi_{\sigma}^{2} d r d t & =\int_{0}^{1} r^{2} z^{1} \phi_{\sigma}(T)-\int_{0}^{1} r^{2} y^{1} \phi_{\sigma}^{0}+\sigma f(0) \int_{0}^{1} r^{2} \phi_{\sigma} \\
& -<z^{0}, \phi_{\sigma, t}(T)>V, V^{\prime}+<y^{0}, \phi_{\sigma}^{1}>V, V^{\prime}
\end{aligned}
$$

and in consequence

$$
\int_{\omega \times(0, T)} r^{2} \phi_{\sigma}^{2} d r d t \leq C_{\sigma}\left(\left\|\left\{\phi_{\sigma}^{0}, \phi_{\sigma}^{1}\right\}\right\|_{L_{\tau}^{2} \times V^{\prime}}+\left\|\left\{\phi_{\sigma}(T), \phi_{\sigma, \mathfrak{l}}(T)\right\}\right\|_{L_{r}^{2} \times V^{\prime}}+C\right)
$$

where the constant $C>0$ is independent of $\sigma$.

Since inequality (25) does not depend on the value of $f(0)$, we can assume that

$$
C<\left\|\left\{\phi_{\sigma}^{0}, \phi_{\sigma}^{1}\right\}\right\|_{L_{r}^{2} \times V^{\prime}}+\left\|\left\{\phi_{\sigma}(T), \phi_{\sigma, \ell}(T)\right\}\right\|_{L_{r}^{2} \times V^{\prime}}
$$

since the contrary will conclude the proof. Then, proceeding as in steps 3 and 4 we easily obtain the uniform bound for $\left\|r y_{\sigma}\right\|_{\infty}$. It remains to prove the observability property (16) and the estimate (24). This will be done in the following section (Proposition 2 and Lemma 4) and the proof of Proposition 1 will be concluded. 


\section{Observability estimates}

The aim of this section is to prove the observability result (16). To prove this estimate we introduce the following class of functions:

$$
\mathcal{P}=\left\{a \text { measurable }: r^{\delta} a(r, t) \in L^{\infty}(\Omega \times(0, T)) \text { for every } \delta>0\right\} .
$$

Observe that, in particular, the function $g$ defined in the previous section belongs to this class.

Remark 3. If $a \in \mathcal{P}$ then $a \in L^{\infty}\left(0, T ; L^{q}(\Omega)\right)$, for every $1 \leq q<\infty$ :

$$
\begin{aligned}
\int_{0}^{1}|a(r, t)|^{q} d r & =\int_{0}^{1} r^{1 / 2} \frac{|a|^{q}}{r^{1 / 2}} \\
& \leq\left\|r^{1 / 2}|a|^{q}(t)\right\|_{L^{\infty}(0,1)}\left\|r^{-1 / 2}\right\|_{L^{1}(0,1)} \\
& =1 / 2\left\|r^{1 / 2 q}|a|(t)\right\|_{L^{\infty}(0,1)}^{q}
\end{aligned}
$$

Therefore

$\|a(t)\|_{L^{q}(0,1)} \leq C\left\|r^{1 / 2 q} a\right\|_{L^{\infty}(\Omega \times(0, T))}$, for almost every $t \in(0, T)$. (30)

Moreover, for every $l_{1} \neq 0$, it is clear that $a(r, t) \in L^{\infty}\left(\left(l_{1}, l_{2}\right) \times\right.$ $(0, T))$ with

$$
\|a\|_{L^{\infty}\left(\left(l_{1}, l_{2}\right) \times(\theta, T)\right)} \leq \frac{\left\|r^{\delta} a(t)\right\|_{L^{\infty}(\Omega \times(0, T))}}{l_{1}^{\phi}}
$$

Let $\Omega=(0,1)$, we consider the following equation with potential $a \in \mathcal{P}$ :

$$
\begin{cases}r^{2} \xi_{t t}-\left(r^{2} \xi_{r}\right)_{r}+r^{2} a(r, t) \xi=0 & \text { in } \Omega \times(0, T) \\ \xi_{r}(0, t)=\xi(1, t)=0 & \text { for } t \in(0, T) \\ \xi(r, 0)=\xi^{0}(r), \xi_{t}(r, 0)=\xi^{1}(r) & \text { in } \Omega .\end{cases}
$$

The main result of this section is the following:

Proposition 2. Let $2>p>1$. If $T>2 \max \left(l_{1}, 1-l_{2}\right)$, then there exist $q=q(p), 2<q<\infty$ and two positive constants $A, B>0$ such that

$$
\left\|\left\{\xi^{0}, \xi^{1}\right\}\right\|_{L_{*}^{\prime} \times V^{\prime}}^{2} \leq A e^{B\left\|r^{1 / 2 q} a\right\|_{\infty}^{1 / p}} \int_{\omega \times(0, T)} r^{2} \xi^{2} d r d t
$$


for every $a(r, t) \in \mathcal{P}$ and for every solution $\xi$ of (32) with initial data $\left\{\xi^{0}, \xi^{1}\right\} \in L_{r}^{2} \times V^{\prime}$ and where \|\|$_{\infty}$ denotes the norm in $L^{\infty}((0, T) \times \Omega)$.

In the proof of Proposition 2 we are going to do the following change of variables:

$$
v=r \xi .
$$

Let $\xi$ be the solution of (32) with $\left\{\xi^{0}, \xi^{1}\right\} \in L_{r}^{2} \times V^{\prime}$. Then $v$ is solution of the following equation:

$$
\begin{cases}v_{t t}-v_{r r}+a(r, t) v=0 & \text { in } \Omega \times(0, T) \\ v(0, t)=v(1, t)=0 & \text { for } t \in(0, T) \\ v(r, 0)=v^{0}(r), v_{t}(r, 0)=v^{1} & \text { in } \Omega\end{cases}
$$

where $v^{0}(r)=r \xi^{0}(r) \in L^{2}(0,1), v^{1}(r)=r \xi^{1}(r) \in H^{-1}(0,1)$. Observe that for every initial data $\left\{v^{0}, v^{1}\right\} \in L^{2}(0,1) \times H^{-1}(0,1)$, (34) has a unique solution for $a \in \mathcal{P}$ and

$$
v \in C\left([0, T] ; L^{2}(0,1)\right) \cap C^{1}\left([0, T] ; H^{-1}(0,1)\right) .
$$

Reciprocally, if $v$ is the solution of (34) with data $v^{0}, v^{1}$, then $\xi=v / r$ is solution of (32) for initial data $\xi^{0}=v^{0} / r, \xi^{1}=v^{1} / r$.

We observe then a biunique correspondance between the solutions of (32) with initial data in $L_{\tau}^{2} \times V^{\prime}$ and the solutions of (34) with initial data in $L^{2}(\Omega) \times H^{-1}(\Omega)$. Then Proposition 2 is equivalent to:

Proposition 3. Let $2>p>1$. If $T>2 \max \left(l_{1}, 1-l_{2}\right)$, then there exist $q=q(p), 2<q<\infty$, and a constant $C>0$ such that

$$
\left\|\left\{v^{0}, v^{1}\right\}\right\|_{L^{2} \times H^{-1}}^{2} \leq C\left(1+\left\|r^{1 / 2 q} a\right\|_{\infty}\right) e^{C\|a\|_{\infty, q}^{1 / p}} \int_{\omega \times(0, T)} v^{2} d r d t
$$

where \|\|$_{\infty, q}$ denotes the norm in $L^{\infty}\left([0, T] ; L^{q}(0,1)\right)$.

For the proof of Proposition 3 we first study the behavior of the energy

$$
\mathcal{E}(t)=\frac{1}{2}\left\{\|v(t)\|_{L^{2}(\Omega)}^{2}+\left\|v_{t}(t)\right\|_{H^{-1}(\Omega)}^{2}\right\}
$$

Where $\|v\|_{H^{-1}(\Omega)}=\left\|\frac{d}{d r}\left[\frac{-d^{2}}{d r^{2}}\right]^{-1} v\right\|_{L^{2}(\Omega)}$ and $\frac{-d^{2}}{d r^{2}}$ denotes the Laplacian with homegeneous Dirichlet conditions.

We have the following estimate. 
Proposition 4. Let $2>p>1$. There exist $q=q(p), 2<q<\infty$ and two positive constants $A, B>0$ such that :

$$
\mathcal{E}(t) \leq A e^{B\|a\|_{\infty, q}^{1 / p} t} \mathcal{E}(0) \quad \forall t \in(0, T)
$$

for every potential a $\in \mathcal{P}$, for every solution of (94).

Let us assume that Proposition 4 hold in order to prove Proposition 3. The proof of this proposition will be given at the end of this section.

\section{Proof of Proposition 3.}

We proceed by steps.

Step 1.

First of all we observe that time-revertibility of the system satisfied by $v$ and Proposition 4 imply that for every $0 \leq t_{1}<t_{2} \leq T$, we have

$$
\begin{gathered}
\left(t_{2}-t_{1}\right)\left[\left\|v^{0}\right\|_{L^{2}(0,1)}^{2}+\left\|v^{1}\right\|_{H^{-1}(0,1)}^{2}\right] \\
\leq A_{2} e^{C\left\|a_{\|}\right\|_{\infty, q}^{1 / p} t_{2}} \int_{t_{1}}^{t_{2}}\left[\|v(t)\|_{L^{2}(0,1)}^{2}+\left\|v_{t}\right\|_{H^{-1}(0,1)}^{2}\right] .
\end{gathered}
$$

Then, it is sufficient to prove the existence of $t_{1}, t_{2} \in[0, T], t_{1}<t_{2}$ and $C>0$ such that

$$
\int_{t_{1}}^{t_{2}}\left[\|v(t)\|_{L^{2}(0,1)}^{2}+\left\|v_{t}\right\|_{H^{-1}(0,1)}^{2}\right] \leq C e^{C\|a\|_{\infty \infty, q}^{1 / p}} \int_{\omega \times(0, T)} v^{2}(r, t) d r d t .
$$

We now observe that, in order to get (38), it is sufficient to prove

$$
\int_{t_{1}}^{t_{2}}\|v(t)\|_{L^{2}(0,1)}^{2} \leq C e^{C\|a\|_{\infty, q}^{1 / p}} \int_{\omega \times(0, T)} v^{2}(r, t) d r d t
$$

Indeed, multiplying (34) by $m(t)\left[\frac{-d^{2}}{d r^{2}}\right]^{-1} v$ and integrating by parts in $\Omega \times\left(t_{1}, t_{2}\right)$ we get

$$
\begin{aligned}
\int_{t_{1}}^{t_{2}} m(t)\left\|v_{t}(t)\right\|_{H^{-1}(0,1)}^{2} & =\int_{t_{1}}^{t_{2}} m(t)\|v(t)\|_{L^{2}(0,1)}^{2}+ \\
& +\int_{\Omega \times\left(t_{1}, t_{2}\right)} m(t) a(r, t) v\left[\frac{-d^{2}}{d r^{2}}\right]^{-1} v_{t} d r d t- \\
& -\int_{t_{1}}^{t_{2}} m^{\prime}(t)<\left[\frac{-d^{2}}{d r^{2}}\right]^{-1} v(t), v_{t}>_{H_{0}^{1}, H^{-1}} d t+ \\
& +\left.\left\{m(t)<\left[\frac{-d^{2}}{d r^{2}}\right]^{-1} v(t), v_{t}>_{H_{0}^{1}, H^{-1}}\right\}\right|_{t_{1}} ^{t_{2}} .
\end{aligned}
$$


Choosing $\left.m \in C^{1}\left(\mid t_{1}, t_{2}\right]\right), m \geq 0$, such that $m\left(t_{1}\right)=m\left(t_{2}\right)=0$,

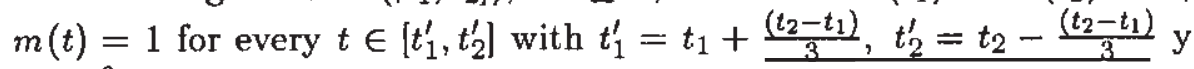
$\frac{\left|m^{\prime}(t)\right|^{2}}{m(t)} \in L^{\infty}\left(t_{1}, t_{2}\right)$ we obtain

$$
\int_{t_{1}^{\prime}}^{t_{2}^{\prime}}\left\|v_{t}\right\|_{H^{-1}(0,1)}^{2} d t \leq C\left(1+\|a\|_{\infty, 2}\right) \int_{t_{1}}^{t_{2}}\|v(t)\|_{L^{2}(\Omega)}^{2} d t
$$

Combining (39) and (40) it is easy to get (38).

\section{Step 2.}

If $v \in L^{2}(\omega \times(0, T))=L^{2}\left(l_{1}, l_{2} ; L^{2}(0, T)\right)$ by using the equation

$$
v_{r r}=v_{t t}+a v
$$

we deduce that $v_{r r} \in L^{2}\left(l_{1}, l_{2} ; H^{-2}(0, T)\right)$ with

$$
\left\|v_{T r}\right\|_{L^{2}\left(l_{1}, l_{2} ; H^{-2}(0 ; T)\right)} \leq C\left(1+\|a\|_{L^{\infty}((0, T) \times \omega)}\right)\|v\|_{L^{2}(\omega \times(0, T))} .
$$

Interpolating (see [6] Vol. I, pp. 12-23), we obtain $v_{r} \in$ $L^{2}\left(l_{1}, l_{2} ; H^{-1}(0, T)\right)$ with

$$
\left\|v_{r}\right\|_{L^{2}\left(l_{1}, l_{2} ; H^{-1}(0, T)\right)} \leq\left(1+\|a\|_{L^{\infty}((0, T) \times \omega)}^{1 / 2}\right)\|v\|_{L^{2}(\omega \times(0, T))}
$$

From (41) we deduce that, in order to get (39) it is sufficient to obtain

$$
\int_{\Omega \times\left(t_{1}, t_{2}\right)} v^{2} d r d t \leq C e^{C\|a\|_{\infty, q}^{1 / p}}\left\{\int_{\omega \times(0, T)} v^{2} d r d t+\int_{l_{1}}^{l_{2}}\left\|v_{r}\right\|_{H^{-1}(0, T)}^{2}\right\}
$$

for some $C>0$. Indeed, combining (42) with (41) we get

$$
\int_{\Omega \times\left(t_{1}, t_{2}\right)} v^{2} d r d t \leq C\left(1+\|a\|_{L^{\infty}((0, T) \times \omega)}\right) e^{C\|a\|_{\infty, q}^{1 / p}} \int_{\omega \times(0, T)} v^{2} d r d t
$$

and in view of $(31)$

$$
\int_{\Omega \times\left(t_{1}, t_{2}\right)} v^{2} d r d t \leq C\left(1+\left\|r^{1 / 2 q} a\right\|_{\infty}^{1 / p)} e^{C\|a\|_{\infty, q}^{1 / p}} \int_{\omega \times(0, T)} v^{2} d r d t .\right.
$$

We observe that in order to prove (42) it is enough to obtain

$$
\int_{\tau\left(r_{0}\right)} v^{2} d r d t \leq C e^{C\|x\|_{\infty, q}^{1 / p}}\left\{\int_{0}^{T} v^{2}\left(r_{0}, t\right) d t+\left\|v_{r}\left(r_{0}, t\right)\right\|_{H^{-1}(0, T)}^{2}\right\}
$$


for every $r_{0} \in \Omega$ and $T>\max \left(r_{0}, 1-r_{0}\right)$ with $\tau\left(r_{0}\right)=\tau_{1}\left(r_{0}\right) \cup \tau_{2}\left(r_{0}\right)$ where

$$
\begin{aligned}
& \tau_{1}\left(r_{0}\right)=\left\{(r, t) \in\left(0, r_{0}\right) \times(0, T): t \in\left(r_{0}-r, r+T-r_{0}\right)\right\} \\
& \tau_{2}\left(r_{0}\right)=\left\{(r, t) \in\left(r_{0}, 1\right) \times(0, T): t \in\left(r-r_{0}, T-r+r_{0}\right)\right\}
\end{aligned}
$$

Indeed, integrating (44) with respect to those $r_{0} \in \omega$ for which the time given in Proposition 2 satisfies $T>2 \max \left(r_{0}, 1-r_{0}\right)$ we get (42) with $t_{1}=\max \left(l_{1}, 1-l_{2}\right), t_{2}=T-\max \left(l_{1}, 1-l_{2}\right)$.

\section{Step 3.}

Let us finally prove (44). We observe that due to finite propagation $(=1)$ in system $(34)$ we have

$$
v=w \text { en } \tau\left(r_{0}\right)
$$

where $w=w(r, t)$ is the solution of

$$
\begin{cases}w_{r r}-w_{t t}-a w=0 & \text { in } \Omega \times(0, T) \\ w(r, 0)=w(r, T)=0 & \text { for every } r \in \Omega \\ w\left(r_{0}, t\right)=v\left(r_{0}, t\right), w_{r}\left(r_{0}, t\right)=v_{t}\left(r_{0}, t\right) & \text { in }(0, T)\end{cases}
$$

System (46) is a wave equation where the roles of the time and space variables have been interchanged. It is an evolution equation with respect to $r$.

We can apply Proposition 4 to system (46). We get

$$
\begin{gathered}
\|w(r)\|_{L^{2}(0, T)}^{2}+\left\|w_{r}(r)\right\|_{H^{-1}(0, T)}^{2} \\
\leq e^{C\|a\|_{\infty, q}^{1 / p} \max \left(r_{0}, 1-r_{0}\right)}\left\{\left\|v\left(r_{0}\right)\right\|_{L^{2}(0, T)}^{2}+\left\|v_{r}\left(r_{0}\right)\right\|_{H^{-1}(0, T)}^{2}\right\}
\end{gathered}
$$

Combining (47) and (45) we get (44).

We proceed now to prove Proposition 4. In this aim we remember two technical Lemmas that are going to be used during the proof.

First we recall the Sobolev's embeddings in dimension one:

Lemma 2. Let $I \subset \mathbb{R}$ be an interval and $0<r \leq s<1,1 \leq p \leq q \leq \infty$. Then

$$
\begin{gathered}
W^{s, p}(I) \subset W^{r, q}(I) \text { if } s-\frac{1}{p}=r-\frac{1}{q} \\
\|f\|_{W^{r, q}(I)} \leq C\|f\|_{W^{s, p}(I)} \quad \forall f \in W^{s, p}(I),
\end{gathered}
$$


with $C=C(r, s)>0$. Moreover,

$$
W^{s, p}(I) \subset L^{q}(I) \text { if } 0<\frac{1}{p}-\frac{1}{q}<s .
$$

For the proof see e.g. [8].

The following result is due to Simon in [9] p. II-15.

Lemma 3. Let $\rho>1, \mathcal{O} \subset \mathbb{R}^{n}$ an open and regular subset. Suppose that

$$
v \in W^{\sigma, r}(\mathcal{O}) \text { where } \sigma \leq \rho, \rho \leq r \leq \infty .
$$

Then

$$
|v|^{\rho-1} v \in W^{\sigma-\delta, r / \rho}(\mathcal{O}) \text { for every } \delta>0
$$

with

$$
\left\|\left.v\right|^{\rho-1} v\right\|_{W^{\sigma-\delta, r / \rho}} \leq C\|v\|_{W^{\sigma, r}}^{\rho} \quad \forall v \in W^{\sigma, r}(\mathcal{O}) .
$$

for a constant $C>0$.

We can take $\delta=0$ if $r=\infty$ or if $\sigma$ is an integer.

We consider the following linear wave equation:

$$
\left\{\begin{array}{l}
y_{t t}-y_{r r}+a y=b \text { in } \Omega \times(0, T) \\
y(0, t)=y(1, t)=0 \text { for } t \in(0, T) \\
y(r, 0)=y^{0}, y_{t}(r, 0)=y^{1} \text { in } \Omega .
\end{array}\right.
$$

with $a \in \mathcal{P}, 1 / 2>\varepsilon \geq 0, b \in L^{2}\left(0, T, H^{-\varepsilon}(\Omega)\right), y \in C\left([0, T] ; H^{1-\varepsilon}(\Omega)\right)$ and $y_{t} \in C\left([0, T] ; H^{\varepsilon}(\Omega)\right)$.

We define the energy:

$$
E(t)=\frac{1}{2}\left\{\|y\|_{H^{1-\varepsilon}}^{2}+\|y t\|_{H^{-\varepsilon}}^{2}\right\},
$$

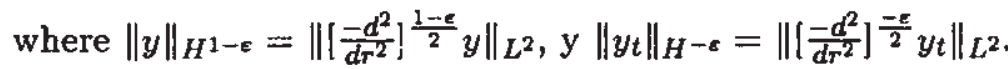

We have the following estimate:

Lemma 4. Let $2>p>1$. If $1 / 2>\varepsilon \geq 0$ is small enough, then there exist $q=q(p), 2<q<\infty$ and constants $A, B>0$ such that

$$
E(t) \leq A\left(E(0)+\|b\|_{L^{2}(0, T ; H-\varepsilon(\Omega))}^{2}\right) e^{B\|a\|_{\infty, q}^{1 / p} t}, \quad \forall t \in(0, T) .
$$


For the proof of Lemma 4 we proceed by steps. First of all we are going to prove the following Lemma:

Lemma 5. Let $\Omega=(0,1)$ and $2<\tilde{q}<3$. There exist $1>\theta>1 / 2$ and $\varepsilon$ small enough such that

$$
W^{1-\varepsilon-\delta, \frac{2}{\bar{q}-1}}(\Omega) \subset W^{\frac{\varepsilon}{1-\theta}+\frac{\bar{q}-2}{(1-\theta) 2 \bar{q}}, \frac{\grave{q}}{\bar{q}-1}}(\Omega)
$$

for some $\delta>0$.

Proof.

We can choose $\theta$ such that

$$
\frac{\tilde{q}-2}{5 \tilde{q}-\tilde{q}^{2}-2}<(1-\theta)<1 / 2
$$

since $2<\tilde{q}<3$ implies

$$
0<\frac{\tilde{q}-2}{5 \tilde{q}-\tilde{q}^{2}-2}<1 / 4
$$

For this election of $\theta$ we have

$$
0<\frac{\tilde{q}-2}{(1-\theta) 2 \tilde{q}}-\frac{\tilde{q}-1}{\bar{q}}+\frac{\tilde{q}-1}{\overline{2}}<1 .
$$

In consequence for $\varepsilon>0$ small enough, $s=\varepsilon+\frac{\varepsilon}{1-0}+\frac{\bar{q}-2}{(1-\theta) 2 \tilde{q}}-\frac{\vec{q}-1}{\tilde{q}}+$ $\frac{\tilde{q}-1}{2}$ satisfies

$$
0<s<1 \text {. }
$$

Let $\delta=1-s$. By Lemma 2 .

$$
W^{1-\varepsilon-\delta, \frac{2}{\bar{q}-1}}(\Omega) \subset W^{\frac{\varepsilon}{1-\theta}+\frac{\bar{q}-2}{(1-\theta) 2 \bar{q}}, \frac{\bar{q}}{\bar{q}-1}}(\Omega) .
$$

\section{Proof of Lemma 4.}

Let us define the perturbed energy

$$
E_{K}(t)=E(t)+\frac{1}{2} K\|y\|_{L^{\bar{q}}}^{2}
$$

where $2<\tilde{q}<3$ is going to be chosen later, $K=\|a\|_{\infty, q}$ and $\frac{1}{\tilde{q}}+\frac{1}{q}=\frac{1}{2}$. 
By Lemma 3 we know that $y^{\bar{q}-1} \in H^{\varepsilon}(\Omega)$ and

$$
\frac{d}{d t}\|y\|_{\tilde{q}}^{2}=\frac{d}{d t}\left(\int_{\Omega} y^{\bar{q}}\right)^{2 / \tilde{q}}=2\|y\|_{\tilde{q}}^{2-\tilde{q}}<y^{\bar{q}-1}, y_{t}>_{H^{\varepsilon}, H^{-\varepsilon}} .
$$

We need to estimate $\left\|y^{\hat{q}-1}\right\|_{H^{\epsilon}}$. In view of Lemmas 3 and 5 we have that for $\varepsilon$ small enough and for a good selection of $\theta$

$$
y^{\tilde{q}-1} \in W^{\frac{\dot{\varepsilon}}{1-\theta}+\frac{\dot{q}-2}{(1-\theta) 2 \dot{q}}, \frac{\dot{q}}{\dot{q}-1}}(\Omega)
$$

Therefore we have that

$$
y^{\tilde{q}-1} \in\left[W^{\frac{\varepsilon}{1-\theta}+\frac{\tilde{q}-2}{(1-\theta) 2 \tilde{q}}, \frac{\tilde{q}}{\tilde{q}-1}}(\Omega), L^{\frac{\tilde{q}}{\dot{q}-1}}(\Omega)\right]_{\theta}=W^{\varepsilon+\frac{\tilde{q}-2}{2 \tilde{q}}, \frac{\tilde{q}}{\bar{q}-1}}(\Omega)
$$

where $[X, Y]_{\theta}$ denotes the interpolation space between the spaces $X$ and $Y$. The equality of these spaces can be found in Grisvard [3].

Moreover, by Lemma 2, we have that

$$
W^{\epsilon+\frac{\bar{q}-2}{2 \bar{q}}, \frac{\bar{q}}{\bar{q}-1}}(\Omega) \subset H^{\varepsilon}(\Omega)
$$

and therefore

$$
\begin{aligned}
& \left\|y^{\bar{q}-1}\right\|_{H^{*}} \leq C\left\|y^{\bar{q}-1}\right\|_{W^{e+\frac{\hat{q}-2}{\partial q}, \frac{\dot{q}}{q-1}}} \\
& \leq C\left\|y^{\bar{q}-1}\right\|_{L}^{\theta}{ }_{L \frac{\bar{q}-1}{\bar{q}-1}}\left\|y^{(\bar{q}-1)},\right\|_{W^{\frac{\varepsilon}{1-\theta}}+\frac{\bar{q}-2}{(1-\theta) 2 \bar{q}}, \overline{\bar{q}}-1}^{(1-\theta)} \\
& \leq C\|y\|_{L \bar{q}}^{(\bar{q}-1) \theta}\|y\|_{H^{1-\epsilon}}^{(\bar{q}-1)(1-\theta)} .
\end{aligned}
$$


Let us multiply (48) by $\left[\frac{-d^{2}}{d r^{2}}\right]^{-\varepsilon} y_{t} \in H^{e}(\Omega)$. Then

$$
\begin{aligned}
\frac{d E_{K}}{d t}= & -\left\langle a(r, t) y,\left[\frac{-d^{2}}{d r^{2}}\right]^{-\varepsilon} y_{t}>_{H^{-\varepsilon}, H^{\varepsilon}}-<b,\left[\frac{-d^{2}}{d r^{2}}\right]^{-\varepsilon} y_{t}>_{H^{-\varepsilon}, H^{\varepsilon}}\right. \\
& +K\|y\|_{L^{\bar{q}}}^{2-\bar{q}}<y^{\bar{q}-1}, y_{t}>_{H^{\varepsilon}, H^{-\varepsilon}} \\
\leq & C\|y\|_{H^{-\varepsilon}}\|a(t)\|_{L^{q}}\|y\|_{L^{\bar{q}}}+\|b\|_{H^{-\varepsilon}}\left\|y_{t}\right\|_{H^{-\varepsilon}} \\
& +K\|y\|_{L^{\bar{q}}}^{2-\bar{q}+\theta(\bar{q}-1)}\|y\|_{H^{1-\varepsilon}}^{(\bar{q}-1)(1-\theta)}\left\|y_{t}\right\|_{H^{-\varepsilon}} . \\
\leq & C K^{1 / 2}\left[\frac{1}{2}\left\|y_{t}\right\|_{H^{-\varepsilon}}^{2}+\frac{K}{2}\left\|y_{L^{\tilde{q}}}^{2}+\frac{K}{2}\right\| y\left\|_{L^{\bar{q}}}^{2(2-\bar{q}+\theta(\bar{q}-1)}\right\| y \|_{H^{-\varepsilon-\varepsilon}}^{2(1-\theta)(\bar{q}-1)}\right. \\
& \left.+\frac{1}{2}\left\|y_{t}\right\|_{H^{-\varepsilon}}^{2}\right]+\frac{1}{2}\|b\|_{H^{-\varepsilon}}^{2}+\frac{1}{2}\left\|y_{t}\right\|_{H^{-\varepsilon}}^{2} \\
\leq & C K^{1 / 2}\left[E_{K}+K^{(\bar{q}-1)(1-\theta)}\left[E_{K}\right]\right]+\frac{1}{2}\|b\|_{H^{-\varepsilon}}^{2}+\frac{1}{2}\left\|y_{t}\right\|_{H^{-\varepsilon}}^{2} \\
\leq & C\left(1+K^{1 / 2+(\bar{q}-1)(1-\theta)}\right) E_{K}(t)+\frac{1}{2}\|b\|_{H^{-\varepsilon}}^{2} .
\end{aligned}
$$

By Gronwall's Lemma we obtain

$$
E_{K}(t) \leq C e^{\gamma t}\left(E_{K}(0)+\|b\|_{L^{2}\left(0, T ; H^{-\varepsilon}\right)}^{2}\right) \text { for every } t \in(0, T)
$$

where $\gamma=C+C\|a\|_{L \omega, q}^{1 / 2+(\tilde{q}-1)(1-\theta)}$.

Now, if we write $1 / p=1 / 2+\beta$ with $1 / 2 \geq \beta>0$, it is clear that if we can choose $\tilde{q}$, such that $\theta$ given by Lemma 5 satisfies $(\tilde{q}-1)(1-\theta)=\beta$ then the proof will be concluded. Let us see that this can be done.

It is enough to see that there exists $\dot{2}<\bar{q}<3$ such that

$$
(1-\theta)=\frac{\beta}{\tilde{q}-1}
$$

satisfies (52). That is, we have to see that for some $3>\tilde{q}>2$ we have that

$$
\beta>\frac{(\tilde{q}-2)(\tilde{q}-1)}{5 \tilde{q}-\tilde{q}^{2}-2}=m(q) .
$$

But we can obtain the last inequality because we have that $\beta>0$ and that $m(\tilde{q})$ is continuous in $\tilde{q}=2$ with $m(2) \cdot=0$. 
Proof of Proposition 4. We write $v=w+y$ where $w$ is the solution of

$$
\left\{\begin{array}{l}
w_{t t}-w_{r r}=0 \text { in } \Omega \times(0, T) \\
w(0, t)=w(1, t)=0 \text { for every } t \in(0, T) \\
w(r, 0)=v^{0}, w_{t}(r, 0)=v^{1} \text { in } \Omega,
\end{array}\right.
$$

and $y$ is the solution of

$$
\left\{\begin{array}{l}
y_{t t}-y_{r r}+a y=-a w \text { in } \Omega \times(0, T) \\
y(0, t)=y(1, t)=0 \text { for every } t \in(0, T) \\
y(r, 0)=0, y_{t}(r, 0)=0 \text { in } \Omega
\end{array}\right.
$$

The energy $\mathcal{E}$ is conserved for system (52). Therefore

$$
\|w(t)\|_{L^{2}(\Omega)}^{2}+\|w(t)\|_{H^{-1}(\Omega)}^{2}=2 \mathcal{E}(0) \quad \forall t \in[0, T] .
$$

We observe that for every $0<\varepsilon<1 / 2$, aw $\in L^{2}\left(0, T ; H^{-\varepsilon}(\Omega)\right)$. In view of Lemma $2, H^{\varepsilon}(\Omega) \subset L^{p}(\Omega)$ for $p=\frac{2}{1-2 \varepsilon}$. Moreover, we know that $a \in L^{\infty}\left(0, T ; L^{2 / \varepsilon}(\Omega)\right)$, in consequence

$$
<a w, \varphi>_{H^{-\varepsilon}, H^{\varepsilon}}=\int_{0}^{1} a w \varphi \leq C\|w\|_{L^{2}}\|a\|_{L^{2 / \epsilon}}\|\varphi\|_{H^{\varepsilon}} \forall \varphi \in H^{\varepsilon}(\Omega),
$$

and then

$$
\|a w\|_{H^{-\varepsilon}} \leq C\|u\|_{L^{2}}\|a\|_{L^{2 / \varepsilon}} .
$$

We apply Lemma 4 with $y^{0}=y^{1}=0$ and $b=-a w$. We get

$$
\begin{aligned}
\|y(t)\|_{H^{1-\varepsilon}}^{2}+\left\|y_{t}(t)\right\|_{H^{-\varepsilon}}^{2} & \leq C\|a\|_{\infty, 2 / \varepsilon}^{2}\|w\|_{L^{2}((0, T) \times \Omega)}^{2} e^{B\|a\|_{\infty, q} t} \\
& \leq 2 C\|a\|_{\infty, 2 / \varepsilon}^{2} \mathcal{E}(0) e^{B\|a\|_{\infty, q}^{1 / p} t}
\end{aligned}
$$

Combining (54) and (55), (36) follows easily.

\section{References}

[1] Bardos, C; Lebeau, G; Rauch, J. [1992] Sharp sufficient conditions for the observation, control and stabilization of waves from the boundary, SIAM J. Control Optim. 30, pp.1024-1065.

[2] Cazenave, T; Haraux, A. [1980] Equations d' évolution avec nonlinéarité logarithmique, Ann. Fac. Sci. Toulouse, 2, pp. 21-51. 
[3] Grisvard, P. [1967] Caractérisation de Quelques Espaces d'Interpolation. Arch. Rat. Mech. Anal. 25 pp. 40-63.

[4] Lions, J.L. [1988] Contrôlabilité exacte, stabilisation et perturbations de systèmes distribués. Tome 1. Contrôlabilité exacte, Masson, RMA8, París.

[5] Lions, J.L. [1988] Exact controllability, stabilization and perturbations for distributed systems, SIAM Rev., 30, pp. 1-68.

[6] Lions, J.L.; Magenes, E. [1968] Problèmes aux limites non homogènes et applications. Vol I y II. Dunod, París.

[7] Simon, J. [1987], Compact sets in the space $\mathbf{L}^{p}(0, T ; B)$. Annali di Matematica pura ed Applicata, (IV), Vol. CXLVI, pp.1173-1191.

[8] Simon, J. [1978?] Fractional Sobolev Theorem in one dimension. Publications du L. A. N., 85023. Université P. et M. Curie. París.

[9] Simon, J.[1977] Sur des equations aux derivées partielles non linéaires Thèse de doctorat d'Etat. Paris 6.

[10] de Teresa, L. [1992] Controlabilidad exacta para las soluciones radiales de la ecuación de ondas semilineal. Trabajo de investigación para créditos de doctorado, UCM.

[11] Zuazua, E. [1993] Exact controllability for semilinear wave equations in one space dimension, Ann. Inst. Henri Poincaré, Vol.10, $n$ 1, pp. 109-129.

[12] Zuazua, E. [1990] An introduction to the exact controllability for distributed systems, Textos e Notas 44, CMAF, Universidades Lisboa.

[13] Zuazua, E. [1990] Contrôlabilité exacte d' une équation des ondes surlinéaire à une dimension d' espace, C.R. Acad. Sci. Paris 311 p.285-290.

[14] Zuazua, E. [1990] Exact Boundary controllability for the semilinear wave equation, in Nonlinear partial differential equations and their applications, H. Brezis y J.L. Lions eds., Séminaire du Collège de 
France 1987/1988, X, Research Notes in Mathematics, Pitman, pp. $357-391$.

IMATE, UNAM;

Circuito Exterior, C.U. 04510, D.F. México.

e-mail: deteresaggauss . matem . unam . mx

Recibido: 17 de Febrero de 1997 